\title{
Specific impairment of visual spatial covert attention mechanisms in Parkinson's disease
}

\author{
Joana Sampaio a , Elzbieta Bobrowicz-Campos a , Rui André ${ }^{a, b}$, Inês Almeida ${ }^{a}$, Pedro Faria ${ }^{a}$, \\ Cristina Januário $^{\mathrm{a}, \mathrm{b}}$, António Freire ${ }^{\mathrm{a}, \mathrm{b}}$, Miguel Castelo-Branco ${ }^{\mathrm{a}, *}$ \\ a Visual Neuroscience Laboratory, IBILI, Faculty of Medicine, University of Coimbra, Azinhaga de Santa Comba, 3000-548 Coimbra, Portugal \\ ${ }^{\mathrm{b}}$ Faculty of Medicine and Coimbra University Hospital, Coimbra, Portugal
}

\section{A R T I C L E I N F O}

\section{Article history:}

Received 17 March 2010

Received in revised form 3 November 2010

Accepted 7 November 2010

Available online 12 November 2010

\section{Keywords:}

Visual impairment

Covert attention

Visual integration

Parkinson's disease

Visual dorsal stream

Parietal cortex

Inter-hemispheric asymmetries

Spatial processing

\begin{abstract}
A B S T R A C T
Visual deficits in early and high level processing nodes have been documented in Parkinson's disease (PD). Non-motor high level visual integration deficits in PD seem to have a cortical basis independently of a low level retinal contribution. It is however an open question whether sensory and visual attention deficits can be separated in PD. Here, we have explicitly separated visual and attentional disease related patterns of performance, by using bias free staircase procedures measuring psychophysical contrast sensitivity across visual space under covert attention conditions with distinct types of cues (valid, neutral and invalid). This further enabled the analysis of patterns of dorsal-ventral (up-down) and physiological inter-hemispheric asymmetries. We have found that under these carefully controlled covert attention conditions PD subjects show impaired psychophysical performance enhancement by valid attentional cues. Interestingly, PD patients also show paradoxically increased visual homogeneity of spatial performance profiles, suggesting flattening of high level modulation of spatial attention. Finally we have found impaired higher level attentional modulation of contrast sensitivity in the visual periphery, where mechanisms of covert attention are at higher demands.

These findings demonstrate a specific loss of attentional mechanisms in PD and a pathological redistribution of spatial mechanisms of covert attention.
\end{abstract}

(c) 2010 Elsevier Ltd. All rights reserved.

\section{Introduction}

There is substantial evidence for non-motor manifestations in Parkinson's disease (Archibald, Clarke, Mosimann, \& Burn, 2009; Bodis-Wollner, 1990, 2003; Bodis-Wollner et al., 1987; Bodis-Wollner \& Tzelepi, 1998; Bodis-Wollner \& Yahr, 1978; Castelo-Branco et al., 2009; Mosimann et al., 2004; Silva et al., 2005; Uc et al., 2005, 2007; Van Asselen \& Castelo-Branco, 2009). Patient studies clearly separating low level sensory and visual spatial attention deficits within the same task are however still lacking. Recent covert attention studies in normal subjects have allowed for the separation of low level sensory processing from the performance enhancement effects of spatial attention (Carrasco, 2006; Pestilli \& Carrasco, 2005). These studies provide a novel methodological opportunity to isolate and distinguish such sources of impairment in patients.

Abbreviations: PD, Parkinson's disease; UPDRS, Unified Parkinson's Disease Rating Scale; CS, contrast sensitivity.

* Corresponding author. Tel.: +351239 480261; fax: +351 239480280.

E-mail address: mcbranco@ibili.uc.pt (M. Castelo-Branco).
The neural basis of visual deficits in PD (Archibald et al., 2009; Bodis-Wollner, 2003; Silva et al., 2005) and the elucidation of which particular processing mechanisms are impaired (e.g., low level contrast detection and/or high level visual attention mechanisms) are crucial issues that can only be addressed if one measures at the same time contrast sensitivity (CS - reciprocal of Threshold measures) with and without manipulation of spatial bias of attention. We have previously demonstrated the advantages of simultaneously studying low and high level visual functions as a way to disentangle the neural origins of visual sensory, perceptual and cognitive deficits (Castelo-Branco et al., 2006, 2007, 2009; Kozak \& Castelo-Branco, 2009). Also, we have previously developed visual CS tasks under conditions that control the spatial distribution of attention (Silva et al., 2008). Adding a cue leads to an asymmetric redistribution of spatial attention. Experimental control is further enhanced by running randomly interleaved psychophysical staircases in space and time, which helps exploring low and high level mechanisms underlying anisotropies in spatial vision (Silva et al., 2008, 2010).

Visual orienting is related to mechanisms of spatial attention in parietal cortex and is often associated with right hemispheric dominance (Davidson \& Hugdahl, 2004; Ivry \& Robertson, 1998). 
The main mechanisms of automatic "exogenous" orienting of spatial attention can be related to inhibition of return (IOR) and automatic orienting. Visual spatial attention can be covertly dissociated from the direction of gaze in a voluntarily driven way, via a mechanism known as "endogenous" attention. This mechanism is in contrast with the above mentioned automatic, stimulus-driven orienting termed “exogenous" attention (Posner \& Cohen, 1984). Cueing paradigms are frequently used to study endogenous and exogenous orienting of attention (Posner \& Cohen, 1984). An important distinguishing factor between the two is the difference in their time-courses. Whereas the effects of endogenous attention require a few hundred milliseconds to fully develop and can be maintained with effort, exogenous attention peaks within 100-120 ms and diminishes rapidly thereafter (Cheal \& Lyon, 1991; Nakayama \& Mackeben, 1989).

To explicitly tackle the question of how attention modulates visual performance, we have focused on paradigms where cueing is known to be facilitatory and not to induce inhibition of return, which refers to the slowing of a response to a target stimulus presented in the same location as a previous stimulus (Klein, 2000). Accordingly, at relatively short (e.g., 150-ms) cue-target stimulus onset asynchronies (SOAs), attentional orienting to targets at cued vs. uncued locations is facilitated, whereas at relatively long SOAs (e.g., beyond $300 \mathrm{~ms}$ ), it is inhibited (Klein, 2000). Reductions in IOR have been argued to reflect impaired inhibitory processes in PD (Poliakoff et al., 2003, but see Grande et al., 2006). Here we were not focused on IOR, which reflects higher level late attentional processing, but rather on cueing with short cue-target SOA to measure early attentional facilitatory/inhibitory effects on visual performance of valid vs. invalid cues (Pestilli \& Carrasco, 2005).

Putative deficits in visual orienting are also relevant in terms of visual performance asymmetries. Sources of performance asymmetries have been documented at different levels of the visual system (nasotemporal at the level of the retina, up-down at level of the retina, lateral geniculate nucleus and early visual cortex and left-right spatial cortical hemispheric asymmetries). Although some of these asymmetries may cancel out, as is the case of monocular nasotemporal asymmetries, in general a physiological/behavioral consequence can be identified (Silva et al., 2010). This is clearly the case concerning binocular left-right asymmetries (Silva et al., 2008). The relation of these functional asymmetries to cell density across retinotopic representations at different levels of the visual system have been addressed in our previous work (Silva et al., 2008, 2010). In brief, sources of spatial asymmetries in performance within the visual field (VF) can be ascribed to lower levels of visual processing, such as occipital cortex or even the retina (Carrasco, Giordano, \& McElree, 2004; Maia-Lopes et al., 2008; Silva et al., 2008). A pattern of up/down visual field asymmetry has been shown to be also present at the level of striate/extrastriate cortices (Maunsell \& Van Essen, 1987).

Probing such anatomic and physiological substrates of attentional and sensory performance may help provide tools to dissect the different visual processing steps that are affected in PD, including visual attention (Kingstone et al., 2002).

In this paper we have followed the seminal work of Carrasco and colleagues that were to first to show a way to separate sensory perception from attentional enhancement of such performance. We have now extended this innovative strategy to patient work on the non-motor cognitive processes that are often impaired in Parkinson disease (Van Asselen et al., 2009; Van Asselen \& Castelo-Branco, 2009). It has indeed been hypothesized that attentional processes are more active in PD patients (Briand, Hening, Poizner, \& Sereno, 2001), raising the question whether this is due to increased facilitation or reduced inhibition.
There is widespread evidence on the relation between spatial hemispheric dominance and the overlap between the attentional and spatial orienting network (Davidson \& Hugdahl, 2004; Ivry \& Robertson, 1998). Orienting of spatial attention can be related to anisotropic patterns of psychophysical performance (Nakayama \& Mackeben, 1989; Silva et al., 2008) but it is important to recognize that cortical contribution to asymmetric visual performance independently of attentional biases has also been recently considered (Carrasco et al., 2004; Carrasco, Talgar, \& Cameron, 2001; Fuller, Rodriguez, \& Carrasco, 2008). This is the case in terms of dorso/ventral (up/down) asymmetries in letter identification (Mackeben, 1999), visual acuity (Altpeter, Mackeben, \& TrauzettelKlosinski, 2000) and attentional conjunctive visual search tasks (He, Cavanagh, \& Intrilligator, 1996). Furthermore, performance on orientation discrimination tasks depends on the up/down target location (Carrasco et al., 2001). The relevance of the functional superiority of the inferior field in primates and humans is also documented by the over-representation of the lower visual field in area MT (Maunsell \& Van Essen, 1987) and in area V6A (Galletti et al., 1999).

Most of the current evidence for functional anisotropies does nevertheless relate to the right hemispheric dominance of spatial attention and to the beneficial effect of covert spatial attention and valid cues in normal subjects (for a review see Carrasco, 2006). Contrast sensitivity represents a basic visual performance dimension where substantial evidence for the role of focused spatial attention on performance improvement has been achieved. Covert attention may be understood as a neural process that enhances the signal (and thereby contrast sensitivity) from a particular part of the sensory scenario. Indeed, in normal subjects, transient covert attention increases contrast sensitivity at the target location with an informative spatial cue (Pestilli \& Carrasco, 2005). Transient covert attention implies visual scanning in the absence of eye movements (prior to planning a saccade or not) and may have both benefits and costs. Accordingly, it may enhance contrast sensitivity at cued locations and impair contrast sensitivity at uncued (neutral) or invalid locations.

Here, we have dissected sensory (using baseline contrast sensitivity), early (superior/inferior) visual patterns of performance and parietal visual attention networks (by assessing left/right performance and validity effects under covert attention conditions) in early stage PD.

Performance was assessed using an achromatic contrast sensitivity task that probed a parvocellular-biased spatiotemporal frequency channel using stationary sinusoidal gratings of intermediate spatial frequency (ISF, Silva et al., 2005, 2008). These patterns were previously shown to also yield hemifield patterns of cortical physiological asymmetry in normal subjects, thereby proving to be adequate for the present study (Silva et al., 2008). Right/left asymmetries are a direct reflection of the hemispheric dominance of the right hemisphere in visuospatial attention, and their study thereby provides, in addition to the effect of peripheral valid, invalid and neutral cues, further clues to the study of attentional deficits in Parkinson's disease. These concepts relate visual transient attention (and orienting) mechanisms with the notion of limited resources and thereby provide an interesting additional paradigm to probe deficits in $\mathrm{PD}$.

In sum, we expected PD patients to show distinct spatial patterns of performance as compared to controls and in particular to show impairment on early facilitation of valid cues. Testing at two distinct eccentricities helped probing the prediction whether more peripheral locations (where attentional demands are higher) show a validity effect as compared to central ones, where attentional demands are lower. We have mainly tested exogenous attention and facilitation, by using a $100 \mathrm{~ms}$ cue-target onset asynchrony. 
Table 1

Subjects characteristics - neuropsychological data.

Patients
(a) $7.13(3.01)$
(b) $5(0.13)^{*}$
(c) $36.13(2.35)^{*}$
(d) $6.93(0.47)$
(e) $5.20(0.26)$
(f) $37.67(3.63)$
(g) $14.00(0.59)$
(h) 73.53 (4.57)

$32.19(3.47)$ (a) $8.00(0.31)$

(b) $5.61(0.21)$

(c) $44.87(2.84)$

(d) $7.09(0.39)$

(e) $5.04(0.21)$

(f) $38.04(3.08)$

(g) $15.22(0.62)$

(h) $82.91(4.92)$

$36.70(3.12)$ Mean (Std. Error)

$100 \%$ right handed

$100 \%$ right handed

Handedness

d.c. - direct condition; i.c. - inverse condition.

${ }^{*} p<0.05$ (this minor difference does not survive correction for multiple comparisons and this score is not correlated with psychophysical measures).

\section{Methods}

\subsection{Participants selection}

35 PD patients (18 males, 17 females) were initially included for this study. The control group comprised 24 demographically matched subjects. Seven patients were excluded due to neuropthalmological exclusion criteria (see below). Given that the main goal of the study was focused on covert attention mechanisms we have further excluded PD patients that were not able to maintain fixation and/or to inhibit reflexive saccades in the presence of exogenous cues $(n=11)$. We discuss below this surprisingly high exclusion rate in terms of the current knowledge of orienting mechanisms in PD. PD patients $(n=17$, after these stringent selection steps) were matched in terms of age and education to controls (Patients: age, $58.12 \pm 7.75$ (mean \pm SD), education, $7.94 \pm 4.83$ years; Controls: age, $54.92 \pm 7.98$, education, $7.75 \pm 4.58$ ). The patients were recruited from the Neurology Department of the Hospital of the University of Coimbra. A complete ophthalmological examination was performed in all individuals including: (1) best-corrected visual acuity (VA-Snellen chart); (2) IOP (intraocular pressure) measurement (Goldman applanation tonometer); (3) slit lamp examination of anterior chamber; (4) angle and fundus examination (Goldman lens). Ophthalmologic exclusion criteria included the following: cataract or other eye disease that might interfere with fundus examination; retinal diseases; neuro-ophthalmologic pathology; and high ammetropy ( sphere $\mathrm{dpt}>4$ and cylinder $\mathrm{dpt}>2$ ).

Informed consent was obtained from all participants. The study was conducted in accordance with the tenets of the Declaration of Helsinki, and with the guidelines of the Ethics Committee of the Faculty of Medicine of the University of Coimbra.

Average daily L-Dopa equivalent dosage was $213.6 \mathrm{mg}$ and motor Unified Parkinson's Disease Rating Scale (UPDRS) 20 mean \pm 9.6 SD. Mean \pm SD Hoehn-Yahr stage and disease duration were $2 \pm 0.63$ and $7.87 \pm 7.13$ years, respectively.

\subsection{Neuropsychological assessment}

Patients with neurological/psychiatric conditions (such as dementia or depression) other than Parkinson's disease were excluded using the Mini Mental State Examination ( $\geq 26$ in all patients, above the Portuguese cut-off) and the Hamilton Depression Rating Scale (cut-off 14). The UPDRS and Hoehn and Yahr staging were also used to measure motor impairment and the severity of the disease (see above).
Verbal intelligence was measured with the Vocabulary subtask of the Wechsler Adult Intelligence Scales (WAIS)-III. The Forward and Backward recall versions of the Corsi Block-Tapping task were used as a measure of spatial working memory. Handedness was defined by using a translated version of the Edinburgh Handedness Inventory. See Table 1 for additional details.

\subsection{Psychophysical task to assess visual attention}

\subsubsection{Apparatus}

The stimuli were generated by means of a video board (Cambridge Visual stimulus Generator, VSG2/5) and displayed on a 20 in. gamma-corrected MitsubishiDiamond Pro 2070 SB monitor $(800 \times 600,100 \mathrm{~Hz})$. Eye positions were continuously monitored by means of the Cambridge Research Systems Video Eyetracker $50 \mathrm{~Hz}$ System (resolution, $0.1^{\circ}$ )

\subsubsection{Stimuli}

The target stimuli were vertical Gabor patches with spatial frequency of $3.5 \mathrm{cpd}$ and were presented within 8 locations organized into two zones (Central Zone 1 and Peripheral Zone 2 ), at each of the four visual quadrants with an eccentricity $5.0^{\circ}$ and $14.9^{\circ}$, respectively. Gabor patch diameters were $2.5^{\circ} / 5.0^{\circ}$ for Zone $1 /$ Zone 2 with standard deviations of $0.6^{\circ} / 1.0^{\circ}$ respectively. The cue for Zone 1 was a $0.3^{\circ}$ diameter dark circle located at a distance of $10.3^{\circ}$ from fixation (not overlapping with the stimulus and just signalling the likely quadrant); a $0.4^{\circ}$ cue at $21.4^{\circ}$ from fixation was used for Zone 2 . The cue appeared either in a quadrant or (in the neutral case) was superimposed on the $2.3^{\circ}$ black fixation point (Fig. 1).

Background luminance was $61.7 \mathrm{~cd} / \mathrm{m}^{2}$ and luminance contrast modulation of the stimuli was manipulated according to the Michelson luminance contrast $(\%)=100 \times\left(L_{\max }-L_{\min }\right) /\left(L_{\max }+L_{\min }\right)$.

\subsubsection{Procedure and design (paradigm)}

The two visual attention tasks were performed separately in Zone 1 and Zone 2. Subjects were instructed to perform a contrast sensitivity task (Castelo-Branco et al., 2006, 2007; Mendes et al., 2005), in a darkened room, with the head stabilized by a chin rest. Viewing was binocular at $36 \mathrm{~cm}$. Observers were instructed to fixate during the experiment, and to report the detection of a peripheral target stimulus using a response box.
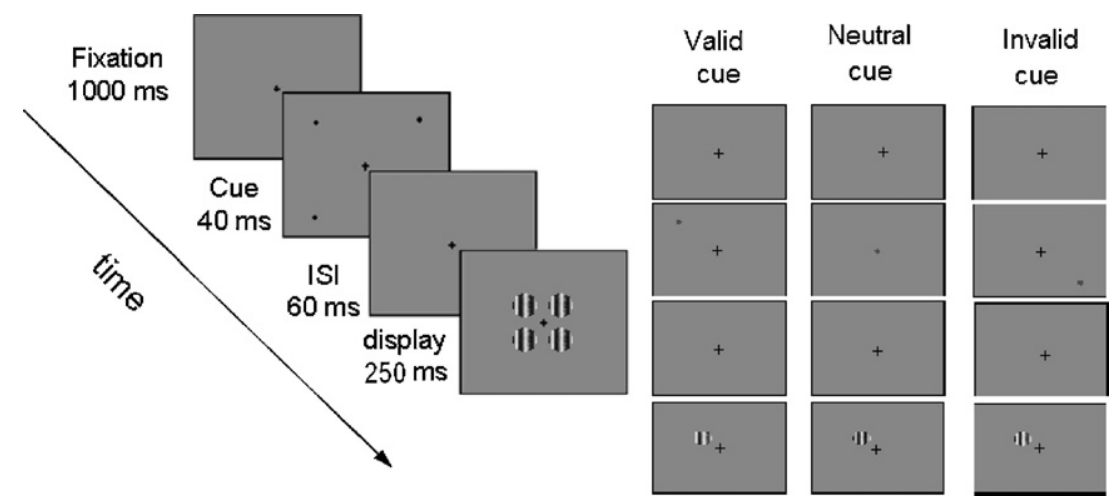

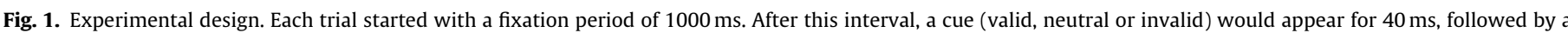
$60 \mathrm{~ms}$ interstimulus interval. The Gabor target stimulus was presented after this interval during $250 \mathrm{~ms}$. 

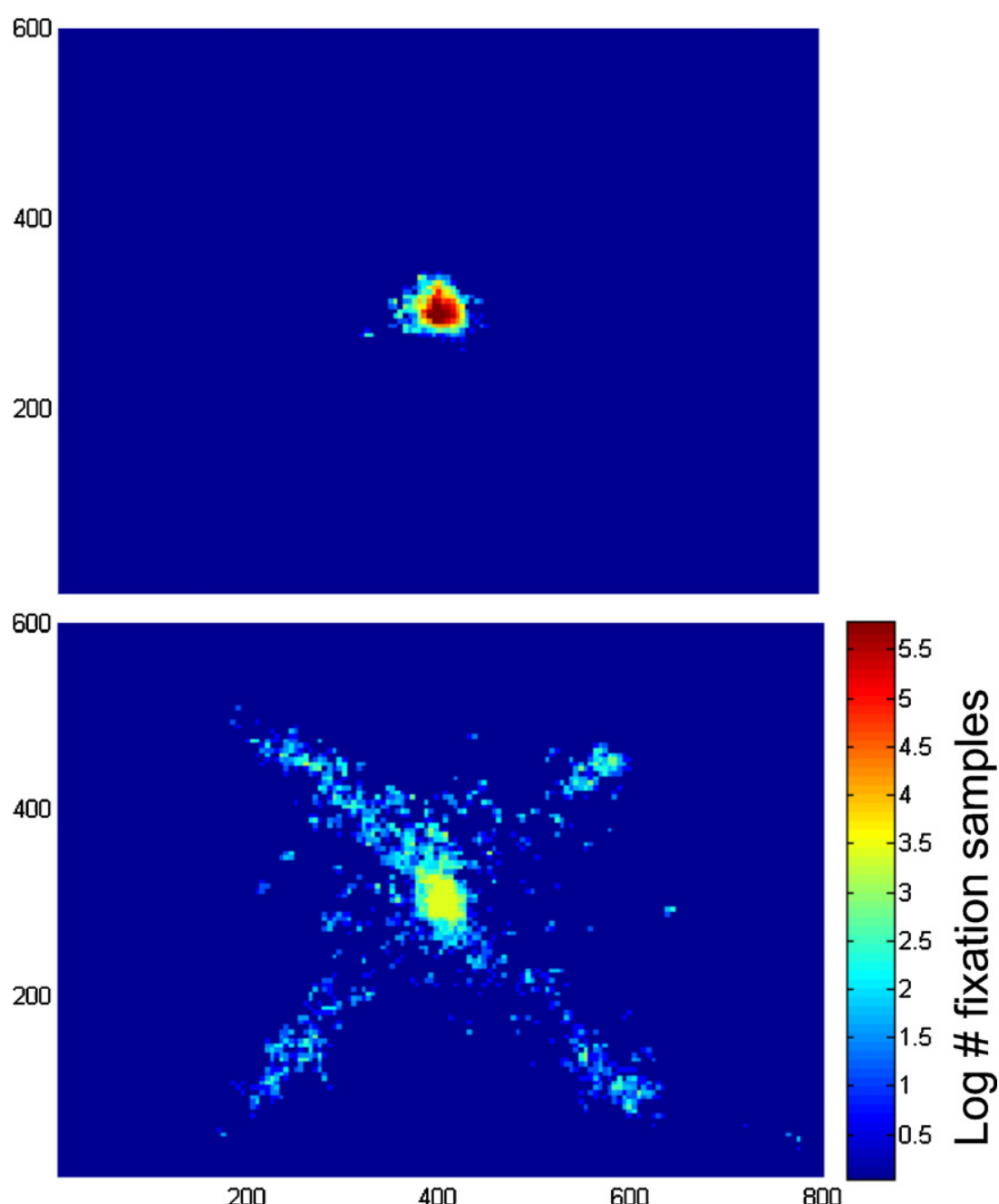

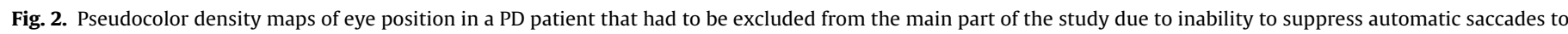

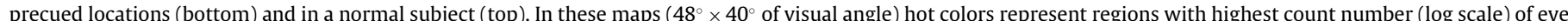

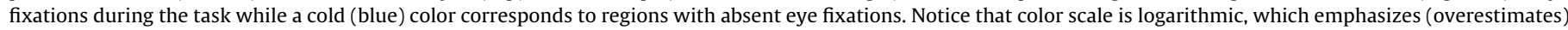

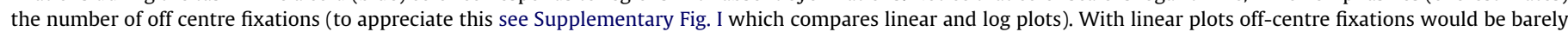
visible because of their much lower number, even if one includes interstimulus interval periods.

Each trial would start with a fixation period of $1000 \mathrm{~ms}$. After this interval, a cue would appear for $40 \mathrm{~ms}$, followed by a $60 \mathrm{~ms}$ interstimulus interval. The Gabor stimulus was presented after this interval during $250 \mathrm{~ms}$.

The cue was equally likely to appear within the centre of the monitor, as a neutral cue (non-informative), or in one of the quadrants. In this last case, the cue could either correctly indicate the quadrant were the stimuli was going to be shown (valid cue), or give a false indication of the Gabor localization (invalid cue). Contrast threshold for each location and each of the 3 cue conditions was estimated by running concomitant randomly interleaved staircases. This allowed for simultaneous determination of contrast sensitivity (reciprocal of \% contrast threshold) across all locations/cue type.

Concerning parameters of our logarithmic staircase, we have used 4 reversals and $15 \%$ of catch trials (positive or negative). A negative catch trial is defined as a corresponding suprathreshold stimulus presentation (maximal contrast) and a positive catch trial corresponds to blank trials. Stimuli (gabor patches) were identical to the ones used in the staircase procedure. We have included neutral/valid/invalid conditions, with a ratio of $1 / 3$ vs. $1 / 3$ vs. $1 / 3$. We have excluded subjects with significant false-positive or false-negative rates $(>33 \%)$. Other staircase parameters: Step Size: $3 \mathrm{~dB}$, Minimum Step Size: 0.1, Initial Value: $50 \%$ contrast Maximum Value: 100\% contrast, Minimum Value: 0, Failures Required to reversal: 1, Successes Required to reversal: 1, Number of Reversals Required to Stop: 4.

We have performed non-parametric statistical analysis on bias effects (false positives and false negatives) and found no significant differences across groups (Mann-Whitney test, both comparisons $n s$ for Zones 1 and 2).

\section{Methodological exclusion of patients unable to keep covert attention}

A substantial proportion of our PD patients showed a surprisingly high tendency to move their eyes towards attentional cues. Our data indicated that pre-selected PD patients have significantly impaired ability to inhibit reflexive saccades when an additional visual pre-cue is added, as compared to controls (Chisquare test, $p<0.01$ ). This difference justified our strict exclusion criteria.

A signature of the loss of inhibitory control typically seen in our PD patients can be observed in Fig. 2. This figure emphasizes offcentre (vs. central) fixations by means of a logarithmic color code (otherwise, the former would be barely visible: see Supplementary Fig. I for comparison of logarithmic vs. linear color codes). One can clearly see that the patient that failed the criterion is often producing eye movements towards the visual pre cues that signal the location of a subsequent target. This lack of inhibitory control of visual orienting is interesting by itself given that it was found in a significant proportion of our early stage PD patients. These patients were for this reason excluded for this study of covert attention 

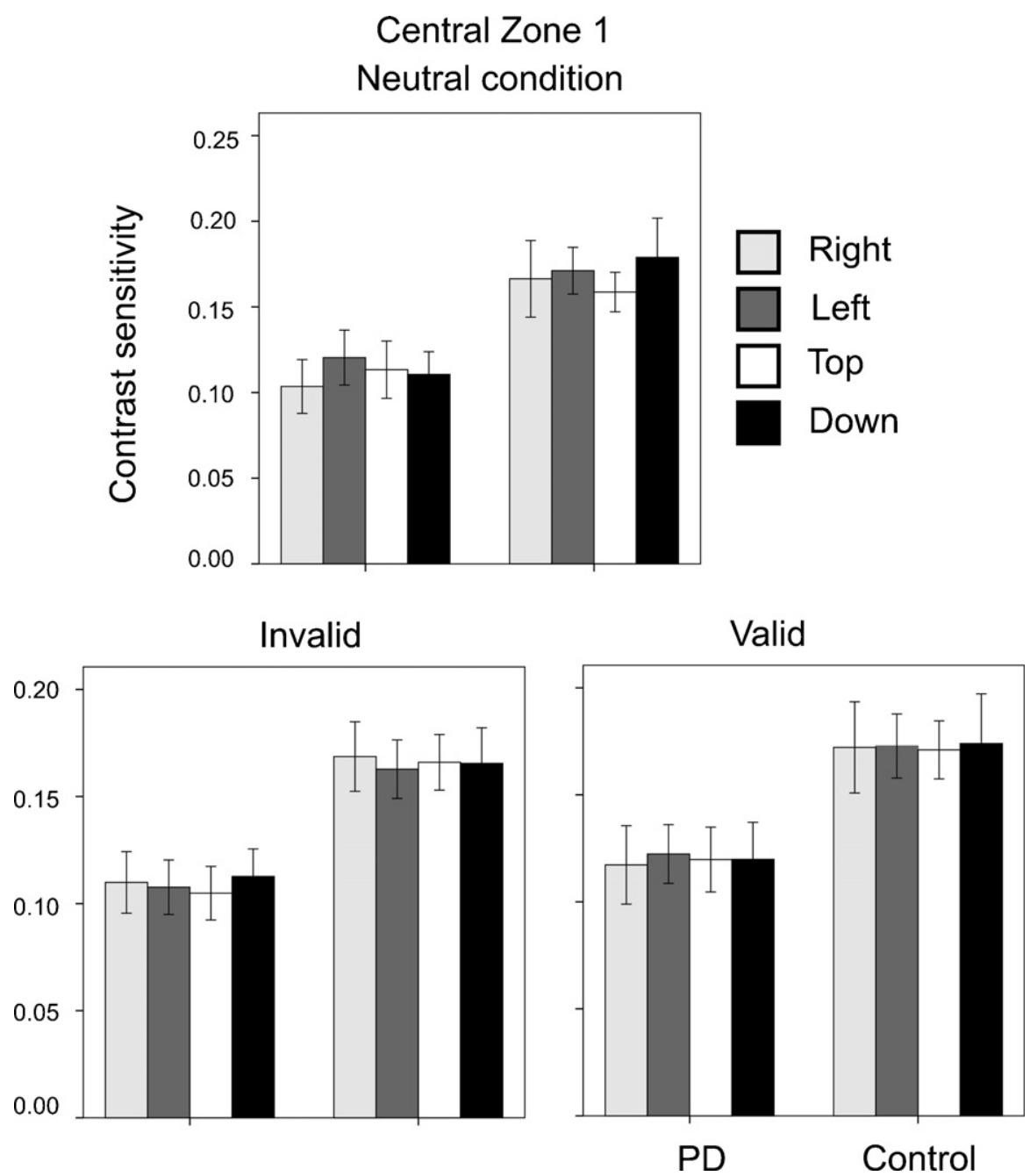

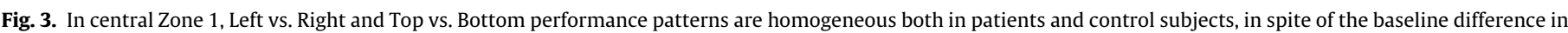
contrast sensitivity (CS). CS is plotted as $1 /(\%$ threshold) here and in subsequent figures.

investigating local contrast sensitivity with or without superimposed attentional spatially distributed signals.

Inclusion criteria for the visual attention tasks were: (a) successfully holding eye fixation on a delimited circular area of $2.5^{\circ}$ at least for $75 \%$ of the test duration, including interstimulus intervals; (b) having a false-positive and false-negative rate under 33\% (by means of presentation of catch trials).

\subsection{Data analysis}

We have chosen to perform parametric analysis (ANOVA analyses of within (e.g. repeated measures) and between-group effects, using SPSS 15) given that data conformed in general to the required assumptions. We have nevertheless adopted the conservative approach of validating our conclusions also with nonparametric analyses. This measure also protected against potential biases introduced by small differences in sample size.

\section{Results}

\subsection{Baseline sensory performance in $P D$}

Concerning central Zone 1 (Fig. 3), we have found a dissociation in performance between controls and PD patients: the pooled contrast sensitivity in Zone 1 was significantly different between groups ( $p=0.01$, ANOVA). Given that the study of main effects "collapses" variables across "post hoc" levels we have also tested whether this difference held true across regions (Fig. 3). Interestingly, this difference was still significant even when the analysis was confined to spatial subregions (left, $p=0.028$; right, $p=0.028$; top, $p=0.007$; bottom, $p=0.032$ ) and most importantly in the neutral condition ( $p=0.02$ for the main effect), suggesting that for the studied spatial frequency (parvocellular-biased, that emphasizes detailed vision) CS deficits are more prominent in Zone 1. Between group comparisons of performance in Zone 2 showed indeed smaller impairment for this specific spatial frequency ( $p=0.056$, for global CS averages, and $p=0.112(n s)$ for the neutral precue, which the critical comparison).

These observations of low level impairment do not mean that the visual periphery is unimpaired or should show less high level attentional modulation of contrast sensitivity. We have in fact proven that attentional/cortical patterns of high level deficits in the visual periphery are stronger in PD. These differences between perifoveal (Zone 1 ) and peripheral (Zone 2 ) were expected because near the fovea it is easier to covertly attend (Van Asselen \& CasteloBranco, 2009), explaining why Zone 1 (perifoveal) does not show any effect aside from the main CS effect. As expected from the previous observations that asymmetries are weak or absent in perifoveal locations (Silva et al., 2008) no significant patterns of cortical asymmetry were observed for central Zone 1 . Moreover significant 


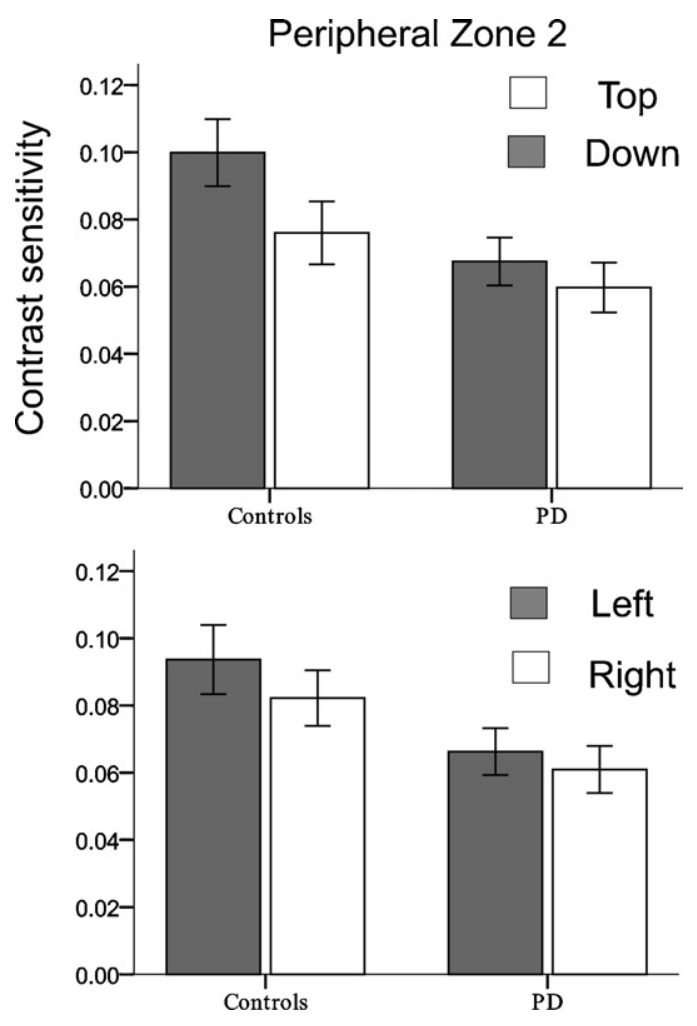

Fig. 4. Flattening of left-right and top-down asymmetry of peripheral (Zone 2) spatial contrast sensitivity under covert attention conditions in PD, in comparison to controls (data pooled across conditions; for analysis split across conditions see text). Planned paired $t$-tests confirmed and replicated (see) left-right and top-down asymmetries in normal subjects (see text), unlike in our PD group. This finding is replicated even when using nonparametric approaches which exclude sample size issues.

effects of the cues were also absent in these perifoveal locations ( $n$ s both the main effect of cue, and also $n s$ for all 3 post hoc comparisons).

\subsection{Patterns of peripheral cortical hemispheric asymmetry differ between PD and normals}

Concerning peripheral Zone 2, within group analysis of patterns of hemispheric and cortical asymmetry revealed a paradoxical increase in spatial homogeneity of performance in the patient group. Indeed, when the neutral cue conditions were assessed significant modulation of left/right and up/down effects were observed only for normal subjects (within group main effect, $p<0.001$, ANOVA repeated measures; post hoc comparisons revealed both top-bottom and left-right asymmetries, $p=0.004$ and $p=0.01$, respectively) and a paradoxical flattening in spatial performance was observed in PD patients (as confirmed by the similar within group and between region explained variance). Fig. 4 depicts the main effect (pooled across conditions) and the same finding (spatial modulation specifically found in controls, Fig. 5) was replicated in the presence of either valid (ANOVA repeated measures main effect, $p=0.004$; post hoc comparisons revealed dominant top-bottom and marginal left-right asymmetries, $p=0.03$ and $p=0.07$, respectively) or invalid cues (ANOVA repeated measures main effect, $p=0.001$, post hoc comparisons revealed dominant top-bottom vs. $n s$ left-right asymmetries in this case ( $p=0.001$ and 0.1 ( $n s)$, respectively).

It is worth emphasizing that the same results were independently replicated three times, regardless of whether analysis was parametric or non-parametric (ruling out sample size issues).

\subsection{Attentional cueing (covert attention) effects on visual performance}

In peripheral contrast sensitivity, allocation of attentional resources to optimize performance is critical. A control condition included a central neutral cue to provide a baseline condition in which spatial attention is not allocated. Within subject analysis showed a significant effect of valid attentional cues $(p=0.03)$ which was specific to the control group, as confirmed by a split-plot analysis of variance (and further validated by non-parametric analysis, as for the other tests). In sum, there was no significant cueing effect for PD.

Under conditions of peripheral cueing, analysis of variance also showed a significant group difference both for valid or invalid conditions (Fig. 6, both between group comparisons with $p=0.03$ and 0.04 , respectively). Importantly, the between group comparison for the neutral precue condition was not significant $(p=0.112)$. Furthermore, there was no within zone PD group effect that could explain the difference. Taken together, these between group results suggest PD patients do not benefit from attentional cues.

\subsection{Correlation with clinical parameters}

We have found no significant correlations between measures of clinical stage (see Section 2 and Table 1 for additional details), such as the motor UPDRS (and Hoehn and Yahr) or disease duration, and contrast sensitivity across distinct locations (central and peripheral), irrespective of the presence/absence of valid/invalid cues, which is in agreement with the fact that our patients were at a relatively early stage. Furthermore, no correlations were observed between contrast sensitivity and performance in the Corsi and vocabulary tasks. Correlations with age were also absent in the patient group.

\section{Discussion}

In this work we were able to identify a specific attentional deficit in PD that could be isolated by explicitly controlling for baseline performance in contrast sensitivity tasks. Our paradigm represents an advantage over traditional paradigms because it does not merely rely on reaction time or percent correct measures which are prone to bias (Fuller et al., 2008). We focused on true psychophysical sensitivity and staircases provide in this respect a great advantage when comparing performance in the presence vs. absence of a spatial cue. Accordingly, psychophysical staircase procedures, target unpredictability and presence of catch trials lead to asymptotic measures of thresholds and minimize variability. Estimates based on \% correct measures showing "large effects" should be taken carefully, and are a potential indication of bias. This is very likely using the "method of constant stimuli", which we have avoided.

We have found that PD patients do not benefit from the effect of attentional cues and show paradoxically flattening of spatial performance profiles. Impaired attentional modulation of contrast sensitivity dominates in the visual periphery, where mechanisms of covert attention are at higher demands. The differences in performance patterns across groups between perifoveal and peripheral regions were expected because near the fovea it is easier to covertly attend (Van Asselen \& Castelo-Branco, 2009).

Although there is evidence for impaired visual speed of processing, as well as general visuospatial and visual search deficits in PD (Bodis-Wollner, 2003; Castelo-Branco et al., 2009; Van Asselen \& Castelo-Branco, 2009; Uc et al., 2005, 2007) no study had attempted before such an explicit dissection between visual 


\section{Peripheral Zone 2}
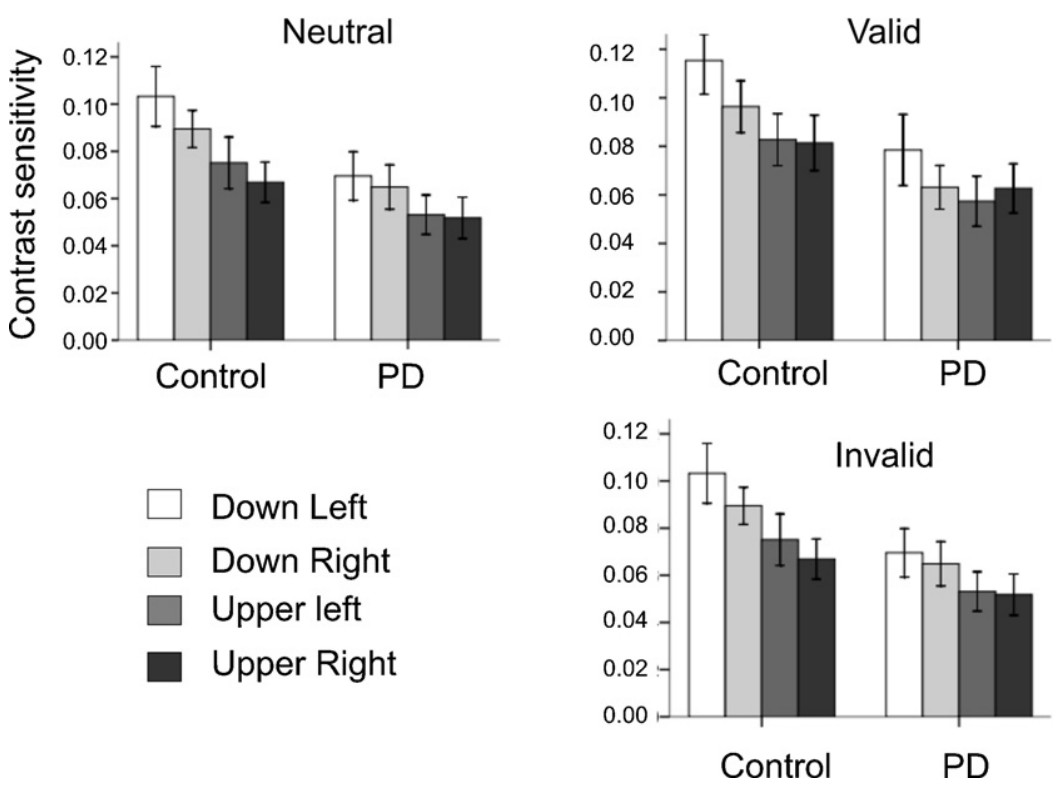

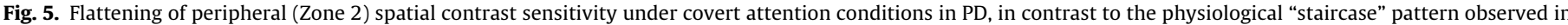

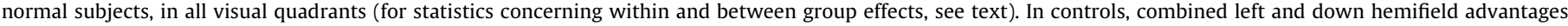
(corresponding to the right hemisphere and dorsal cortex) lead to a quadrant wise pattern of performance that is absent in patients.

sensitivity and spatial attention within the same task, thereby separating low level visual and high level spatial vision attentional processes.

One outstanding difficulty in isolating attentional deficits concerns distinguishing perceptual bias vs. real performance impairment. The mere use of simple suprathreshold paradigms (Caffarra, Riggio, Malvezzi, Scaglioni, \& Freedman, 1997; Hsieh, Hwang, Tsai, \& Tsai, 1996), in which subjects are instructed to respond by pressing a response key after the appearance of a suprathreshold target may be misleading because no thresholds for target detectability are being measured. Such a strategy precludes the study of the effects of attention on visual performance. In addition, confounds related to subjective preference for a given spatial location (choice bias) dominates with suprathreshold stimuli. This may preclude otherwise detectable impairment of task-related distribution of attentional resources in PD (Bennett, Waterman, Scarpa, \& Castiello, 1995). In our study we were able to fully explore this issue by combining a difficult contrast threshold task (validated

Peripheral Zone $2 \quad \square \quad$ Valid
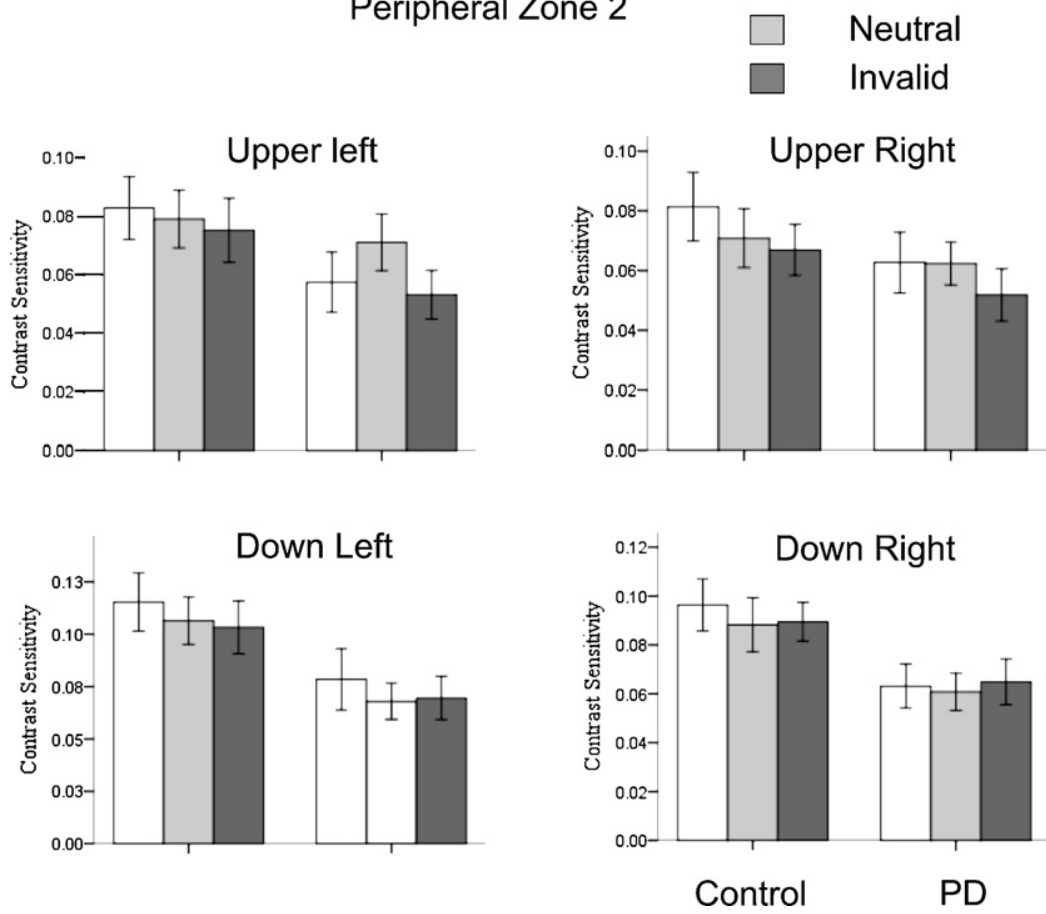

Fig. 6. Comparison of performance for Neutral, Valid and Invalid precue conditions split across visual quadrants. 
by Silva et al., 2008) with a covert attention task with valid/invalid cues.

We found that PD patients lack the beneficial effect of valid cues and there was no within zone PD group effect that could explain the difference with controls. Covert attention mechanisms are therefore ineffective, confirming the suggestion of Yamada, Izyuuinn, Schulzer, and Hirayama (1990) that covert shifts of attention are weak in PD. We show that the beneficial effect of valid cues is independent from a low level visual deficit.

Importantly, we were able to study covert attention mechanisms by excluding PD patients lacking reflexive saccadic inhibition, leading to abnormal automatic orienting to target precues. We had, in fact, to exclude a large number of patients with such loss of automatic response inhibition. Our task design, which enabled to study the role of attentional modulation on a matched low level baseline task, prevented a possible role for cognitive confounds explaining our results. This was further confirmed by the lack of correlation with spatial working memory performance. The strategy followed here to isolate early attentional mechanisms in spatial vision, did not address inhibition of return (Klein, 2000) which reflects higher level late attentional processing.

The above mentioned deficits in visual attention mechanisms are consistent with the loss of both the spatial attention right hemispheric dominance and the visual field inferior-superior performance anisotropy (Silva et al., 2008) and a paradoxical flattening (abnormal redistribution of spatial vision mechanisms) of psychophysical performance profiles in PD. Future studies should address the issue whether such redistribution is sex dependent, although recent evidence suggests that gender explains a very small variance component of these effects (Boles, 2005).

In conclusion, we have found evidence for independent damage of specialized visual spatial attention systems in PD in addition to the low level non-motor deficits that are due to impairment at the level of the retina and early sensory cortex.

\section{Acknowledgements}

This research was funded by grants from the Portuguese Science and Technology Foundation (FCT): PTDC_SAU_NEU_68483_2006, PTDC_PSI_67381_2006 and PIC_IC_82986_2007, as well as by the National Brain Imaging Network of Portugal (BIN).

\section{Appendix A. Supplementary data}

Supplementary data associated with this article can be found, in the online version, at doi:10.1016/j.neuropsychologia.2010.11.002.

\section{References}

Altpeter, E., Mackeben, M., \& Trauzettel-Klosinski, S. (2000). The importance of sustained attention for patients with maculopathies. Vision Research, 40, 1539-1547.

Archibald, N. K., Clarke, M. P., Mosimann, U. P., \& Burn, D. J. (2009). The retina in Parkinson's disease. Brain, 132(Pt 5), 1128-1145.

Bennett, K. M., Waterman, C., Scarpa, M., \& Castiello, U. (1995, February). Covert visuospatial attentional mechanisms in Parkinson's disease. Brain, 118(Pt 1) 153-166.

Bodis-Wollner, I. (1990). Visual deficits related to dopamine deficiency in experimental animals and Parkinson's disease patients. Trends in Neuroscience, 13, 296-302.

Bodis-Wollner, I. (2003). Neuropsychological and perceptual defects in Parkinson's disease. Parkinsonism and Related Disorders, 9, 83-89.

Bodis-Wollner, I., Marx, M. S., Mitra, S., Bobak, P., Mylin, L., \& Yahr, M. (1987). Visual dysfunction in Parkinson's disease. Loss in spatiotemporal contrast sensitivity. Brain, 110, 1675-1698.

Bodis-Wollner, I., \& Tzelepi, A. (1998). The push-pull action of dopamine on spatial tuning of the monkey retina: The effects of dopaminergic deficiency and selective D1 and D2 receptor ligands on the pattern electroretinogram. Vision Research, 38, 1479-1487.

Bodis-Wollner, I., \& Yahr, M. D. (1978). Measurements of visual evoked potentials in Parkinson's disease. Brain, 101, 661-671.
Boles, D. B. (2005). A large-sample study of sex differences in functional cerebral lateralization. Journal of Clinical and Experimental Neuropsychology, 27(6), 759-768. Briand, K. A., Hening, W., Poizner, H., \& Sereno, A. B. (2001). Automatic orienting of visuospatial attention in Parkinson's disease. Neuropsychologia, 39(11), 1240-1249.

Caffarra, P., Riggio, L., Malvezzi, L., Scaglioni, A., \& Freedman, M. (1997). Orienting of visual attention in Alzheimer's disease: Its implication in favor of the interhemispheric balance. Neuropsychiatry and Neuropsychology Behavioral Neurology, 10(2), 90-95.

Carrasco, M. (2006). Covert attention increases contrast sensitivity: Psychophysical, neurophysiological and neuroimaging studies. Progress in Brain Research, 154, 33-70.

Carrasco, M., Giordano, A. M., \& McElree, B. (2004). Temporal performance fields: Visual and attentional factors. Vision Research, 44, 1351-1365.

Carrasco, M., Talgar, C. P., \& Cameron, E. L. (2001). Characterizing visual performance fields: Effects of transient covert attention, spatial frequency, eccentricity, task and set size. Spatial Vision, 15, 61-75.

Castelo-Branco, M., Mendes, M., Silva, M. F., Januário, C., Machado, E., Pinto, A. et al. (2006). Specific retinotopically based magnocellular impairment in a patient with medial visual dorsal stream damage. Neuropsychologia, 44(2), 238253.

Castelo-Branco, M., Mendes, M., Sebastião, A. R., Reis, A., Soares, M., Saraiva, J., et al. (2007, December). Visual phenotype in Williams-Beuren syndrome challenges magnocellular theories explaining human neurodevelopmental visual cortical disorders. The Journal of Clinical Investigation, 117(12), 3720-3729.

Castelo-Branco, M., Mendes, M., Silva, F., Massano, J., Januário, G., Januário, C., et al. (2009, January). Motion integration deficits are independent of magnocellular impairment in Parkinson's disease. Neuropsychologia, 47(2), 314-320.

Cheal, M., \& Lyon, D. R. (1991). Central and peripheral precuing of forced-choice discrimination. Quarterly Journal of Experimental Psychology A: Human Experimental Psychology, 43, 859-880.

Davidson, R. J., \& Hugdahl, K. (Eds.). (2004). The asymmetrical brain. MIT Press.

Fuller, S., Rodriguez, R. Z., \& Carrasco, M. (2008). Apparent contrast differs across the vertical meridian: Visual and attentional factors. The Journal of Vision, 8(1), 16.1-16.

Galletti, C., Fattori, P., Kutz, D. F., \& Gamberini, M. (2000, February). Brain location and visual topography of cortical area V6A in the macaque monkey. European Journal of Neuroscience, 11(2), 575-582.

Grande, L. J., Crosson, B., Heilman, K. M., Bauer, R. M., Kilduff, P., \& McGlinchey, R. E. (2006, May). Visual selective attention in Parkinson's disease: Dissociation of exogenous and endogenous inhibition. Neuropsychology, 20(3), 370-382.

He, S., Cavanagh, P., \& Intrilligator, J. (1996). Attentional resolution and the locus of visual awareness. Nature, 383, 334-337.

Hsieh, S., Hwang, W. J., Tsai, J. J., \& Tsai, C. Y. (1996, June). Visuospatial orienting of attention in Parkinson's disease. Perceptual and Motor Skills, 82(3 Pt 2), 1307-1315.

Ivry, R., \& Robertson, L. C. (1998). The two sides of perception. Cambridge: MIT Press, Series in Cognitive Neurosciences.

Kingstone, A., Klein, R., Morein-Zamir, S., Hunt, A., Fisk, J., \& Maxner, C. (2002, October). Orienting attention in aging and Parkinson's disease: Distinguishing modes of control. Journal of Clinical and Experimental Neuropsychology, 24(7), 951-967.

Klein, R. M. (2000, April). Inhibition of return. Trends in Cognitive Sciences, 4(4), $138-147$.

Kozak, L. R., \& Castelo-Branco, M. (2009, February). Peripheral influences on motion integration in foveal vision are modulated by central local ambiguity and centersurround congruence. Investigative Ophthalmology and Visual Science, 50(2), 980-988 (Epub October 24, 2008)

Mackeben, M. (1999). Sustained focal attention and peripheral letter recognition. Spatial Vision, 12, 51-72.

Maia-Lopes, S., Silva, E. D., Silva, M. F., Reis, A., Faria, P., \& Castelo-Branco, M. (2008, March). Evidence of widespread retinal dysfunction in patients with stargardt disease and morphologically unaffected carrier relatives. Investigative Ophthalmology and Visual Science, 49(3), 1191-1199.

Maunsell, J. H. R., \& Van Essen, D. C. (1987). The topographic organization of the middle temporal visual area in the macaque monkey: Representational biases and relationship to callosal connections and myeloarchitectonic boundaries. The Journal of Comparative Neurology, 266, 535-555.

Mendes, M., Silva, F., Simões, L., Jorge, M., Saraiva, J., \& Castelo-Branco, M. (2005, December). Visual magnocellular and structure from motion perceptual deficits in neurodevelopmental model of dorsal stream function. Brain Research Cognitive Brain Research, 25(3), 788-798.

Mosimann, U. P., Mather, G., Wesnes, K. A., O’Brien, J. T., Burn, D. J., \& McKeith, I. G. (2004). Visual perception in Parkinson disease dementia and dementia with Lewy bodies. Neurology, 63(11), 2091-2096.

Nakayama, K., \& Mackeben, M. (1989). Sustained and transient components of focal visual attention. Vision Research, 29, 1631-1646.

Pestilli, F., \& Carrasco, M. (2005). Attention enhances contrast sensitivity at cued and impairs it at uncued locations. Vision Research, 45(14), 1867-1875.

Poliakoff, E., O’Boyle, D. J., Moore, A. P., McGlone, F. P., Cody, F. W., \& Spence, C. (2003, September). Orienting of attention and Parkinson's disease: Tactile inhibition of return and response inhibition. Brain, 126(Pt 9), 2081-2092 (Epub July 22, 2003).

Posner, M. I., \& Cohen, Y. (1984). Components of visual orienting. In H. Bouma, \& D. Bouwhuis (Eds.), Attention and performance (pp. 531-556). Erlbaum.

Silva, M. F., Faria, P., Regateiro, F. S., Forjaz, V., Januário, C., Freire, A., et al. (2005, October). Independent patterns of damage within magno-, parvo- and koniocellular pathways in Parkinson's disease. Brain, 128(Pt 10), 2260-2271. 
Silva, M. F., Maia-Lopes, S., Mateus, C., Guerreiro, M., Sampaio, J., Faria, P., et al. (2008, January). Retinal and cortical patterns of spatial anisotropy in contrast sensitivity tasks. Vision Research, 48(1), 127-135.

Silva, M. F., Mateus, C., Reis, A., Nunes, S., Fonseca, P., \& Castelo-Branco, M. (2010). Asymmetry of visual sensory mechanisms: Electrophysiological, structural, and psychophysical evidences. Journal of Vision, 10(6), 26.

Uc, E. Y., Rizzo, M., Anderson, S. W., Qian, S., Rodnitzky, R. L., \& Dawson, J. D. (2005). Visual dysfunction in Parkinson disease without dementia. Neurology, 65(12), 1907-1913.

Uc, E. Y., Rizzo, M., Anderson, S. W., Sparks, J. D., Rodnitzky, R. L., \& Dawson, J. D. (2007). Impaired navigation in drivers with Parkinson's disease. Brain, 130(Pt 9), 2433-2440.
Van Asselen, M., Almeida, I., Andre, R., Januário, C., Gonçalves, A. F., \& CasteloBranco, M. (2009, April). The role of the basal ganglia in implicit contextual learning: A study of Parkinson's disease. Neuropsychologia, 47(5), 126912673.

Van Asselen, M, \& Castelo-Branco, M. (2009, January). The role of peripheral vision in implicit contextual cuing. Attention, Perception and Psychophysics, 71(1), $76-81$.

Yamada, T., Izyuuinn, M., Schulzer, M., \& Hirayama, K. (1990, July). Covert orienting attention in Parkinson's disease. Journal of Neurology Neurosurgery and Psychiatry, 53(7), 593-596. 